\title{
QUADRATIC PARTITIONS: PAPER III
}

BY E. T. BELL

1. Simple Summation Formulas. This note is independent of partitions. It gives the general summation formulas of which a very special case might have been used to pass directly from $\S 3$ to $\$ 4$ of the preceding note, ${ }^{*}$ and which will be used in future. The final formulas considerably extend and generalize many in the literature of the Bernoullian and allied functions.

Let $f(x)$ be an entire function of $x$. Write $M \equiv[(n-1) / 2]$, $N \equiv[n / 2]$;

$$
\begin{aligned}
& f_{\beta}(n) \equiv \sum_{r=1}^{M} f(2 r-1+e(n)), \quad f_{\gamma}(n) \equiv \sum_{r=1}^{M}(-1)^{r} f(2 r-1+e(n)), \\
& f_{\eta}(n) \equiv \sum_{r=1}^{N}(-1)^{r} f(2 r-e(n)), f_{\rho}(n) \equiv \sum_{r=1}^{N} f(2 r-e(n)) .
\end{aligned}
$$

One of the pairs $(\beta, \eta),(\rho, \gamma)$ is sufficient, since

$$
\begin{aligned}
f_{\rho}(2 n-1)=f_{\beta}(2 n) & =\sum_{r=1}^{n-1} f(2 r), \\
f_{\rho}(2 n-2)=f_{\beta}(2 n-1) & =\sum_{r=1}^{n-1} f(2 r-1), \\
f_{\gamma}(2 n-1)=f_{\eta}(2 n-2) & =\sum_{r=1}^{n-1}(-1)^{r} f(2 r-1), \\
f_{\gamma}(2 n)=f_{\eta}(2 n-1) & =\sum_{r=1}^{n-1}(-1)^{r} f(2 r) .
\end{aligned}
$$

The like applies to the more general sums $f_{\xi p s}(n)$ in $\S 2$.

It is required to express $f_{\xi}(n)(\xi=\beta, \gamma, \eta, \rho)$ as explicit functions of $n$. Write

$$
\begin{aligned}
C_{\beta}(n) \equiv & 2 e(n) f(0)+2 f(n)+2 e(n)\left\{f\left(2 B^{\prime}\right)-f\left(-2 B^{\prime}\right)\right\} \\
& \cdot 2\{1-e(n)\}\left\{f\left(R^{\prime}\right)-f\left(-R^{\prime}\right)\right\},
\end{aligned}
$$

* This Bulletin, this issue (vol. 38, No. 8), pp. 551-554. The notation and definitions are given in I, ibid., vol. 37 (1931), pp. 870-875. 


$$
\begin{aligned}
C_{\gamma}(n) \equiv & 4 e(n) f(0)-4(-1)^{M} f(n)+2\{1-e(n)\}\{f(E)+f(-E)\} \\
& +2(-1)^{e(n)} e(n)\left\{f\left(2 G^{\prime}\right)-f\left(-2 G^{\prime}\right)\right\} \\
C_{r^{\prime}}(n) \equiv & 2\{1-e(n)\} f(0)+e(n)\{f(E)+f(-E)\} \\
& -\{1-e(n)\}\left\{f\left(2 G^{\prime}\right)-f\left(-2 G^{\prime}\right)\right\}, \\
C_{\rho}(n) \equiv & \{1-e(n)\} f(0)+e(n)\left\{f\left(R^{\prime}\right)-f\left(-R^{\prime}\right)\right\} \\
& +\{1-e(n)\}\left\{f\left(2 B^{\prime}\right)-f\left(-2 B^{\prime}\right)\right\} .
\end{aligned}
$$

Then the required expressions are given by

$$
\begin{aligned}
& 4 f_{\beta}(n)=f\left(\beta^{\prime}(n)\right)-C_{\beta}(n), \quad 8 f_{\gamma}(n)=(-1)^{M} f\left(\gamma^{\prime}(n)\right)-C_{\gamma}(n), \\
& 4 f_{\eta}(n)=(-1)^{N} f(\eta(n))-C_{\eta}(n), \quad 2 f_{\rho}(n)=f\left(\rho^{\prime}(n)\right)-C_{\rho}(n) .
\end{aligned}
$$

It will be sufficient to indicate the genesis of one, say the last. We have (see I, $\$ \S \$ 3,6,7$ ),

$$
\begin{aligned}
\cos \rho^{\prime}(n) x= & 1-e(n)+2 \sum_{r=1}^{N} \cos (2 r-e(n)) x, \\
\sin \rho^{\prime}(n) x= & 2 e(n) \sin R^{\prime} x+2\{1-e(n)\} \sin 2 B^{\prime} x \\
& +2 \sum_{r=1}^{N} \sin (2 r-e(n)) x,
\end{aligned}
$$

from which we pass to a single identity between umbral exponentials on multiplying the second by $i$ and adding to the first. Thence the stated formula for $2 f_{\rho}(n)$ is written down. For, if $a, b, \cdots, c$ denote umbrae for the moment, and $A, B, \cdots, C$, $x$ ordinaries, and if $A \exp a x+B \exp b x+\cdots+C \exp c x \equiv 0$ is an identity in $x$, then $A f(a)+B f(b)+\cdots+C f(c)=0$. This also sufficiently indicates the proofs for the formulas in $\$ 2$, which are obtained from the exponential identities just used on changing therein $x$ into $p x$ and multiplying throughout by exp $s x$.

2. Extended Simple Formulas. The arguments of $f$ in the preceding formulas are in arithmetical progression with common difference 1 . The following refer to any arithmetical progression. Let $p, s$ be ordinates, and write

$$
{ }_{p_{s}}(n) \equiv \sum_{r=1}^{M} f(p(2 r-1+e(n))+s),
$$




$$
\begin{aligned}
& f_{\gamma p s}(n) \equiv \sum_{r=1}^{M}(-1)^{r} f(p(2 r-1+e(n))+s), \\
& f_{\eta p s}(n) \equiv \sum_{r=1}^{N}(-1)^{r} f(p(2 r-e(n))+s), \\
& f_{\rho p s}(n) \equiv \sum_{r=1}^{N} f(p(2 r-e(n))+s) .
\end{aligned}
$$

These include those in $\$ 1: f_{\xi 10}(n)=f_{\xi}(n)$.

Denote by $C_{\xi p s}(n)$ the result of performing the substitution $x \rightarrow p x+s$ on the argument of $f(x)$ in $C_{\xi}(n)$. For example,

$$
\begin{aligned}
C_{\rho p s}(n) \equiv & \{1-e(n)\} f(s)+e(n)\left\{f\left(p R^{\prime}+s\right)-f\left(-p R^{\prime}+s\right)\right\} \\
& +\{1-e(n)\}\left\{f\left(2 p B^{\prime}+s\right)-f\left(-2 p B^{\prime}+s\right)\right\} .
\end{aligned}
$$

Then the expression of $f_{\xi p s}(n)$ as an explicit function of $n$ is obtained from that for $f_{\xi}(n)$ by performing the same substitution on the argument $\xi^{\prime}(n)$ of $f\left(\xi^{\prime}(n)\right)$ and replacing $C_{\xi}(n)$ by $C_{\xi p s}(n)$. For example,

$$
2 f_{\rho p s}(n)=f\left(p \rho^{\prime}(n)+s\right)-C_{\rho p s}(n) .
$$

3. Multiple Summation Formulas. Let $f\left(x_{1}, \cdots, x_{j}\right), t>1$, be an entire function of $\left(x_{1}, \cdots, x_{j}\right)$. We write $f\left(x_{1}, \cdots, x_{t}\right)$ as a purely symbolic or ideal product of $t$ functions $f^{j}\left(x_{j}\right)$ ( $j$ superscript) of one argument, $f\left(x_{1}, \cdots, x_{j}\right) \equiv f^{1}\left(x_{1}\right) \cdots f^{1}\left(x_{j}\right)$. By definition, in this multiplication, $f_{i j}\left(x_{j_{1}}\right) \cdots f_{i j}\left(x_{j_{t}}\right) \equiv f^{1}\left(x_{1}\right) \cdots f^{t}\left(x_{j}\right)$, where $j_{1}, \cdots, j_{t}$ is any permutation of $1, \cdots, t$.

Write $M_{j} \equiv\left[\left(n_{j}-1\right) / 2\right]$, and let $p_{j}, s$, be ordinaries. Then the expression of

$2^{2 t} \sum_{r_{1}=1}^{M_{1}} \cdots \sum_{r_{t}=1}^{M_{t}} f\left(p_{1}\left(2 r_{1}-e\left(n_{1}\right)\right)+s_{1}, \cdots, p_{t}\left(2 r_{t}-e\left(n_{t}\right)\right)+s_{t}\right)$

as an explicit function of $n_{1}, \cdots, n_{t}$ is

$$
\prod_{j=1}^{t}\left[f^{j}\left(p_{j} \beta^{\prime}\left(n_{j}\right)+s_{j}\right)-C_{\beta p_{j s} j}^{j}\left(n_{j}\right)\right]
$$

in which $C_{\beta p j^{s} j}^{j}\left(n_{j}\right)$ is written down from $\$ 2$ by giving to each $f$ in $C_{\beta p_{j} s_{j}}\left(n_{j}\right)$ as there defined the superscript $j$. The indicated product is distributed precisely as in a commutative ring; symbolic products of $f^{j}$ 's are then replaced by their equivalent functions of $t$ arguments by the above definition. 
To validate the process, proceed from $t=2$ by mathematical induction. For $t=2$, we consider $f\left(x_{1}, x_{2}\right)$ as a function of $x_{1}$, keep $x_{2}$ fixed, and apply the summation formulas of $\S 2$. In each term of the result we then consider $f\left(x_{1}, x_{2}\right)$ as a function of $x_{2}$ and apply $\S 2$.

In exactly the same way multiple summations equivalent to symbolic products (as above) of any number of factors of one or more of the types giving the explicit forms of the function $f_{\xi p s}(n),(\xi=\beta, \gamma, \eta, \rho)$, in $\S 2$ can be written out as functions of the upper limits of the summations.

By the method of proof in $\$ \$ 1,2$, it follows that these formulas remain true under linear transformations of the arguments of the entire functions. The like does not hold for non-linear transformations, as the product of two or more umbrae is undefined.

California Institute of Technology

\section{A LOGICAL EXPANSION IN MATHEMATICS*}

\section{BY HASSLER WHITNEY $\dagger$}

1. Introduction. Suppose we have a finite set of objects, (for instance, books on a table), each of which either has or has not a certain given property $A$ (say of being red). Let $n$, or $n(1)$, be the total number of objects, $n(A)$ the number with the property $A$, and $n(\bar{A})$ the number without the property $A$ (with the property not- $A$ or $\bar{A})$. Then obviously

$$
n(\bar{A})=n-n(A) .
$$

Similarly, if $n(A B)$ denote the number with both properties $A$ and $B$, and $n(\bar{A} \bar{B})$ the number with neither property, that is, with both properties not- $A$ and not- $B$, then

$$
n(\bar{A} \bar{B})=n-n(A)-n(B)+n(A B)
$$

which is easily seen to be true.

The extension of these formulas to the general case where any number of properties are considered is quite simple, and is well

* Presented to the Society, October 25, 1930.

$\dagger$ National Research Fellow. 